\title{
ORIGINAL ARTICLE RNA-binding motif protein 47 inhibits Nrf2 activity to suppress tumor growth in lung adenocarcinoma
}

\author{
T Sakurai $^{1}$, K Isogaya ${ }^{1}$, S Sakai ${ }^{1}$, M Morikawa ${ }^{2}$, Y Morishita ${ }^{1}$, S Ehata ${ }^{1}$, K Miyazono ${ }^{1}$ and D Koinuma ${ }^{1}$
}

\begin{abstract}
RNA-binding proteins provide a new layer of posttranscriptional regulation of RNA during cancer progression. We identified RNA-binding motif protein 47 (RBM47) as a target gene of transforming growth factor (TGF)- $\beta$ in mammary gland epithelial cells (NMuMG cells) that have undergone the epithelial-to-mesenchymal transition. TGF- $\beta$ repressed RBM47 expression in NMuMG cells and lung cancer cell lines. Expression of RBM47 correlated with good prognosis in patients with lung, breast and gastric cancer. RBM47 suppressed the expression of cell metabolism-related genes, which were the direct targets of nuclear factor erythroid 2-related factor 2 (Nrf2; also known as NFE2L2). RBM47 bound to KEAP1 and Cullin 3 mRNAs, and knockdown of RBM47 inhibited their protein expression, which led to enhanced binding of Nrf2 to target genomic regions. Knockdown of RBM47 also enhanced the expression of some Nrf2 activators, p21/CDKN1A and MafK induced by TGF- $\beta$. Both mitochondrial respiration rates and the side population cells in lung cancer cells increased in the absence of RBM47. Our findings, together with the enhanced tumor formation and metastasis of xenografted mice by knockdown of the RBM47 expression, suggested tumor-suppressive roles for RBM47 through the inhibition of Nrf2 activity.
\end{abstract}

Oncogene (2016) 35, 5000-5009; doi:10.1038/onc.2016.35; published online 29 February 2016

\section{INTRODUCTION}

Nuclear factor erythroid 2-related factor 2 (Nrf2, also called NFE2L2) is activated by oxidative stress and electrophiles. In normal cells, production of antioxidant proteins through the stabilization of Nrf2 is central to defense against oxidative stress. In non-oxidative conditions, an E3 ubiquitin ligase complex consisting of KEAP1, Cullin 3 (CUL3) and RBX1 immediately ubiquitinates and degrades the Nrf2 protein after synthesis. ${ }^{1}$ In contrast, cancer cells frequently have mutations in NRF2/NFE2L2 and/or KEAP1 genes that result in the stabilization and constitutive activation of the Nrf2 protein. Stabilized Nrf2 heterodimerizes with small Maf family transcription factors and binds to the antioxidant-responsive element in the genome. Nrf2 then induces resistance to oxidative stress or anticancer therapy by activating transcription of its target genes. ${ }^{2,3}$ It has also been reported that cyclin-dependent kinase inhibitor p21 protein (CDKN1A) competes with KEAP1 for Nrf2 binding, ${ }^{4}$ and TGF- $\beta$ promotes heterogeneity and drug resistance of squamous cell carcinoma of the skin through CDKN1A induction. ${ }^{5}$ Nrf2 also changes the cellular metabolism in proliferating cells through the transcriptional regulation of related enzymes. ${ }^{6}$ Therefore, cancers with high Nrf2 levels are associated with poor prognosis because of high proliferation as a result of altered metabolism, as well as resistance to chemotherapy and radiotherapy. ${ }^{7}$

In contrast, transforming growth factor- $\beta$ (TGF- $\beta$ ) is well known to exhibit bidirectional functions in cancer progression; although suppressing tumor growth in the early stage of cancer, it drives cancer progression during the advanced stage. ${ }^{8}$ Importantly, these complex roles of TGF- $\beta$ and downstream Smad and non-Smad signaling pathways in cancer are sometimes attributed to their effects on, or crosstalk with, other signaling pathways. In this study, we searched for RNA-binding proteins that are regulated during the process of epithelial-to-mesenchymal transition by TGF- $\beta$, and identified RNA-binding motif protein 47 (RBM47). Our findings regarding the roles of RBM47 in lung cancer cells harboring a KEAP1 mutation reveal that it acts as a tumor suppressor by controlling constitutive Nrf2 activity as a last resort.

\section{RESULTS}

Identification of RBM47 as a cancer-related target of TGF- $\beta$ We analyzed our published RNA-sequencing (seq) data from mouse mammary epithelial NMuMG cells in which $24 \mathrm{~h}$ of TGF- $\beta$ treatment recapitulated several features observed in advanced breast cancer cells, such as epithelial-to-mesenchymal transition. ${ }^{9}$ Of the 653 RNA-binding proteins, $\mathrm{Rbm} 47$ was the most strongly downregulated gene by TGF- $\beta$ (Supplementary Table S1). RBM47 is highly conserved among rat, mouse, orangutan and dog (Figure 1a) and reportedly localizes mainly in the nucleus; ${ }^{10,11}$ however, we found that both endogenous and exogenous RBM47 were expressed in the cytoplasm and the nucleus (Figure $1 \mathrm{~b}$ and Supplementary Figure S1). Quantitative reverse transcription-polymerase chain reaction ( $q R T-P C R$ ) analysis of 22 cell lines revealed that RBM47 was expressed in cell lines from lung adenocarcinoma, breast cancer and gastric cancer (Figure 1c). We then used the collection of published microarray data of several types of cancers and found that high expression of RBM47 correlated significantly with good prognosis of patients (Figure 1d). ${ }^{12,13}$ Multivariate analysis using Cox proportional hazard regression model was available in lung cancer data (histology and stage as prognostic factors) and breast cancer data (ER and HER2 expressions as prognostic factors), and expression of RBM47 was an independent prognostic factor in lung cancer

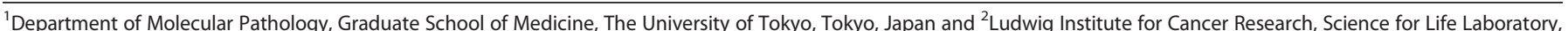

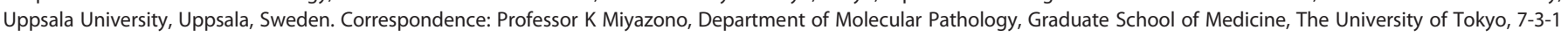
Hongo, Bunkyo-ku, Tokyo 113 0033, Japan.

E-mail: miyazono@m.u-tokyo.ac.jp

Received 16 September 2015; revised 22 December 2015; accepted 11 January 2016; published online 29 February 2016
} 
a

\begin{tabular}{l|c|c|c|c|c|c|}
\hline Human & & RRM1 & RRM2 & RRM3 & & Alanine-rich \\
\hline \multirow{2}{*}{ Rat } & $90 \%$ & $99 \%$ & $98 \%$ & $97 \%$ & $94 \%$ & $91 \%$ \\
\hline \multirow{2}{*}{ Mouse } & $87 \%$ & $99 \%$ & $98 \%$ & $99 \%$ & $94 \%$ & $95 \%$ \\
\hline \multirow{nyyyyyy}{*}{ Orangutan } & $97 \%$ & $100 \%$ & $100 \%$ & $100 \%$ & $100 \%$ & $99 \%$ \\
\hline
\end{tabular}

b

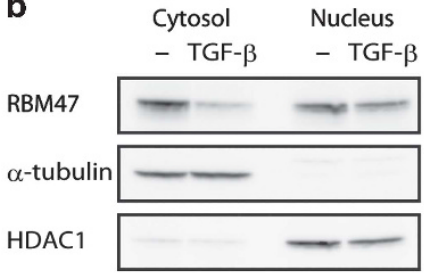

C

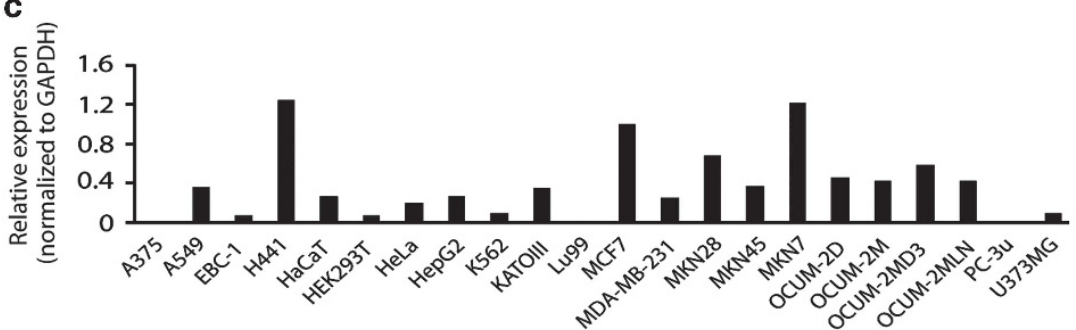

d
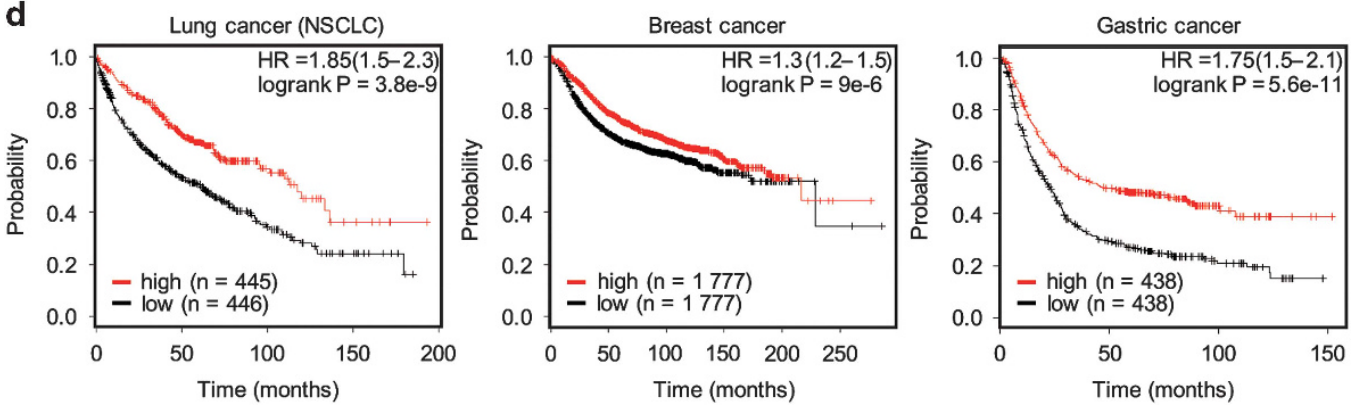

Figure 1. Reduced expression of RBM47 is associated with poor outcomes for lung, breast and gastric cancer patients. (a) A scheme of RBM47 protein with interspecies amino acid conservation. RRM1-3, RNA recognition motifs 1-3. (b) The subcellular localization of endogenous RBM47 protein after treatment of H441 cells with $2.5 \mathrm{ng} / \mathrm{ml}$ TGF- $\beta$ for $24 \mathrm{~h}$ was determined by immunoblotting. (c) The levels of RBM47 expression in a panel of 22 cell lines were examined using qRT-PCR and normalized with respect to GAPDH expression. (d) Survival rates based on RBM47 expression were analyzed by Kaplan-Meier survival method in lung (overall survival), breast (relapse-free survival) and gastric cancer (overall survival) patients. The half of patients with higher expression of RBM47 mRNA is indicated in red and that with lower expression is indicated in black. A log-rank test was used to compare the variance between the two groups.

patients $(P=0.0127$, hazard ratio $=1.37$ (confidence interval: $1.08-1.75)$ ) but not in breast cancer patients $(P=0.0813$, hazard ratio $=1.11(0.99$ 1.27)). We therefore focused on the role of RBM47 in lung cancer cells.

Transcriptional regulation of RBM47 by TGF- $\beta$ and TTF-1 qRT-PCR and immunoblot analyses revealed that TGF- $\beta$ downregulated the expression of RBM47 mRNA and protein in these cells (Figure $1 \mathrm{~b}$ and Figures $2 \mathrm{a}$ and $\mathrm{b}$ ). Thyroid transcription factor 1 (TTF-1, also known as NKX2-1) is expressed in lung adenocarcinoma, ${ }^{14}$ and high TTF-1 expression is in general associated with good prognosis. TTF-1 antagonizes TGF- $\beta$ signaling via disruption of the Smad3Smad4 complex in the nucleus, which leads to inhibition of the epithelial-to-mesenchymal transition process induced by TGF- $\beta .^{15,16}$ On the basis of our published chromatin immunoprecipitationsequencing (ChIP-seq) data using anti-TTF-1 antibody in H441 cells, ${ }^{15}$ we identified significant TTF-1-binding peaks at the RBM47 locus (Figure 2c). Moreover, Smad3 binding to the RBM47 locus was upregulated by TTF-1 siRNA (siTTF-1) in the Smad3 ChIP-seq data (Figure 2c), which was accompanied by decreased RBM47 protein in H441 cells (Figure 2d). We also prepared a promoter-reporter construct that contained a genomic sequence near the transcription start site of RBM47 $(-1950 \mathrm{bp} \sim+50 \mathrm{bp})$. We found that RBM47 promoter activity was increased by forced expression of TTF-1 (Figure 2e) and decreased by TTF-1 siRNAs (siTTF-1\#1, \#2) (Figure 2f). In contrast, RBM47 promoter activity was decreased by TGF- $\beta$, which was partially canceled by Smad3 siRNAs
(siSmad3\#1, \#2) (Figure 2g). These results suggest that TTF-1 and Smad3 bind to the RBM47 promoter, and either maintain or suppress RBM47 expression, respectively.

RBM47 inhibits Nrf2 activity through several regulatory molecules We established A549-Luc cells in which luciferase was constitutively expressed and RBM47 expression was stably suppressed by short hairpin RNAs (shRNAs) against RBM47 (A549-Luc-shRBM47\#1, \#2) (Figure 3a). A549-Luc cells stably expressing control shRNA (A549-Luc-shCT) were also established. We performed RNA-sequencing (seq) and focused on RNAs whose fragments per kilobases of exon per million sequence read values were equal or more than 10 in any of the samples. Gene set enrichment analysis revealed that the direct target genes of Nrf2 were the second most enriched oncogenic signature and were upregulated by shRBM47 (Supplementary Table S2 and Figure $3 \mathrm{~b}) .{ }^{17}$ These Nrf2 target genes included aldo-keto reductase family 1 , member $C 1$ (AKR1C1), aldehyde dehydrogenase 3 family, member A1 (ALDH3A1), glutathione peroxidase 2 (GPX2) and sulfiredoxin 1 (SRXN1) (Supplementary Table S3). Their expression levels were upregulated in A549-Luc-shRBM47 cells, as confirmed by qRT-PCR (Figure 3c). Therefore, we focused on the Nrf2 pathway in the present study.

We extracted endogenous RBM47-bound RNAs from the total RNA of A549 cells by RNA immunoprecipitation (RIP). Then, we quantified RIP samples by deep sequencing (RIP-seq). Given the 

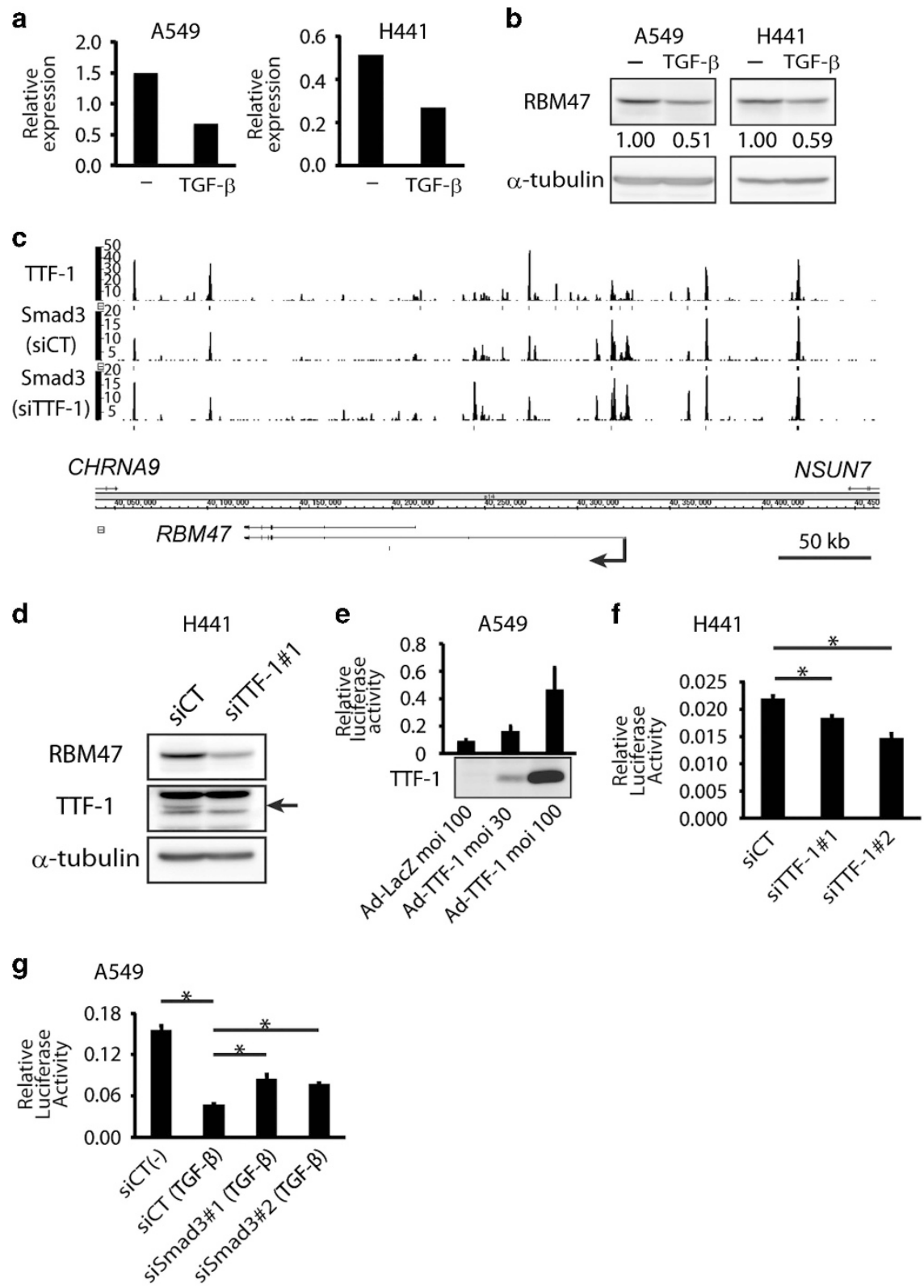

Figure 2. Regulation of RBM47 by TGF- $\beta$ and TTF- 1 in lung adenocarcinoma cells. (a, b) After the $24-h$ treatment of A549 cells and H441 cells with $2.5 \mathrm{ng} / \mathrm{ml}$ TGF- $\beta$, expression levels of RBM47 mRNA and protein were examined by qRT-PCR (a) and immunoblot (b) analyses, respectively. $\alpha$-tubulin was used as a loading control in (b). Protein expression was quantified using Image $J$ and normalized to that of lane 1 and is indicated below each panel. (c) ChIP-seq data of Smad3 and TTF-1 binding at the RBM47 locus in H441 cells. An arrow indicates the transcription starting site of RBM47. TTF-1 binding (top panel) and Smad3 binding (center and bottom panels) in TGF- $\beta$-treated H441 cells transfected with control or TTF-1 siRNAs, respectively, are shown. (d) H441 cells transfected with control or TTF-1 siRNAs were examined by immunoblot analysis to evaluate the effect of TTF-1 on the expression of RBM47 protein. The arrow indicates the endogenous TTF-1 protein. (e) A549 cells were transfected with human RBM47 promoter-reporter construct in combination with adenoviral FLAG-TTF-1 or LacZ infection. At $48 \mathrm{~h}$ after infection, cells were harvested and assayed for luciferase activities. Averages and standard deviations of the two biological replicates were shown for each condition. Expression of FLAG-TTF-1 was confirmed by immunoblotting (bottom panel). (f) H441 cells were transfected with human RBM47 promoterreporter construct in combination with control or TTF-1 siRNAs (siTTF-1\#1 and siTTF-1\#2). At $48 \mathrm{~h}$ after transfection, cells were harvested and assayed for luciferase activities. siCT: control siRNA. Averages and standard deviations of the two biological replicates were shown for each condition. ${ }^{*} P<0.01$. (g) A549 cells were transfected with human RBM47 promoter-reporter construct in combination with control or Smad3 siRNAs (siSmad3\#1 and siSmad3\#2). At $24 \mathrm{~h}$ after transfection, cells were treated with $2.5 \mathrm{ng} / \mathrm{ml}$ TGF- $\beta$ for $24 \mathrm{~h}$ and harvested for luciferase activities. Averages and standard deviations of the two technical replicates were shown for each condition. ${ }^{*} P<0.01$.

list of RBM47-bound RNAs (Supplementary Table S4), we searched for Nrf2 pathway genes and found that RBM47 bound to KEAP1 and CUL3 mRNAs (Figure 3d). Decreased expression of KEAP1 and CUL3 proteins in A549-Luc-shRBM47 cells was observed (Figure 3e). Notably, we did not find any changes in the expression of Nrf2 protein (Supplementary Figure S2a) and nuclear import of
Nrf2 (Supplementary Figure S2b). Moreover, we did not find any changes in the half-life of KEAP1 and CUL3 mRNAs (Supplementary Figure S2c) by shRBM47. KEAP1 and CUL3 mRNAs were rather increased in shRBM47 cells (Figure 3f). These findings suggest that RBM47 binds to KEAP1 and CUL3 mRNA and upregulates their protein expression levels. A549 cells have a homozygous mutation 
a

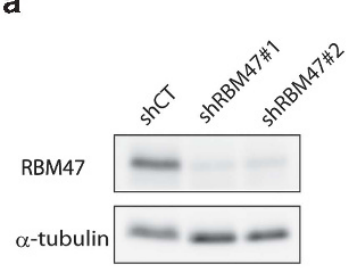

C
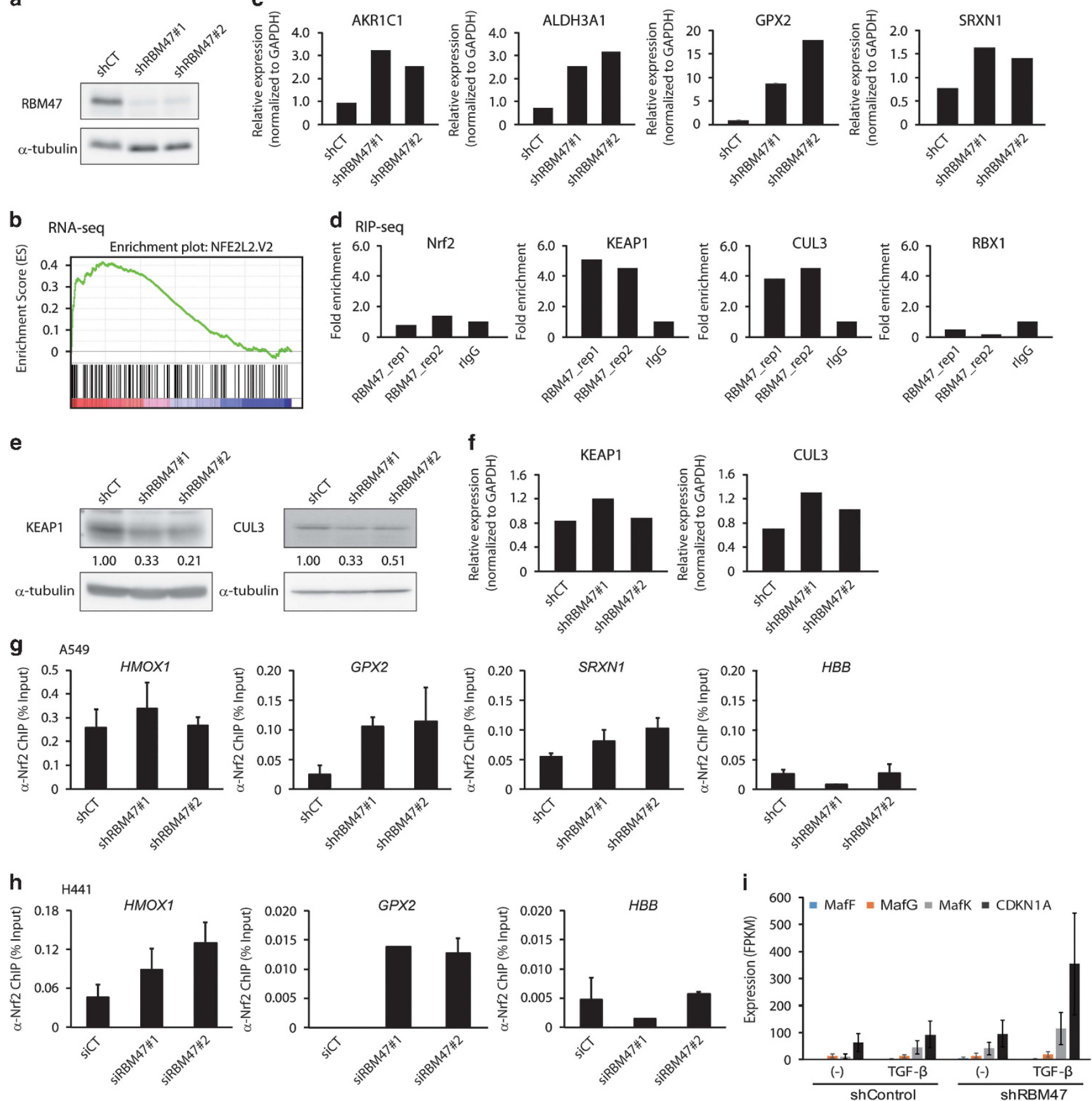

Figure 3. RNA-seq and RIP-seq analyses reveal that knockdown of RBM47 elevates Nrf2 activity. (a) A549-Luc cells were infected with lentiviral vector encoding control shRNA (shCT) or shRNAs against RBM47 (shRBM47\#1 and \#2). RBM47 expression was examined in A549-Luc-shCT cells and A549-Luc-shRBM47 cells by immunoblot analysis. (b) Enrichment of Nrf2 targets in the list of genes that were upregulated by knockdown of RBM47. Gene expression data obtained by RNA-seq in A549-Luc-shCT cells and A549-Luc-shRBM47\#1 cells were used for gene set enrichment analysis. A gene set NFE2L2.V2 of c6 MSigDB oncogenic signature (v4.0) consisting of the direct gene targets of Nrf2 was identified as the second most enriched phenotype of the analysis; its enrichment plot is shown. Normalized enrichment score (NES) $=1.854$. Expression data of the genes whose expression levels exceeded 10 fragments per kilobases of exon per million sequence read (FPKM) in any of the samples were used for the analysis. (c) qRT-PCR analysis of the identified Nrf2 target genes regulated by RBM47. (d) RIP-seq data of Nrf2 and its regulatory genes. RIP-seq was performed in A549 cells using anti-RBM47 or normal rabbit lgG (rlgG) and SNRNP70 as a control. RBM47_rep1 and RBM47_rep2 represent biological replicates of anti-RBM47 RIP. Quantified FPKM values of RBM47 RIP samples were normalized against rlgG-RIP-seq FPKM. (e) Immunoblotting of KEAP1 and CUL3 proteins in A549-Luc cells expressing shCT or shRBM47s. Protein expression was quantified using Image $J$ and normalized to that of lane 1 and is indicated below each panel. (f) qRT-PCR analysis of KEAP1 and CUL3 expression in cells after RBM47 knockdown. (g) A quantitative ChIP-PCR analysis of Nrf2 binding at its target-binding regions in A549 cells. HBB: control genomic region. (h) qChIP-PCR analysis was performed in H441 cells after knockdown of RBM47 expression by siRNAs (siRBM47\#1 and siRBM47\#2). siCT: control siRNA. In (g) and (h), averages and standard deviations of the three technical replicates were shown for each condition. (i) Expression of small Maf family and CDKN1A was determined by RNA-seq. Error bars: $95 \%$ confidence interval calculated by Cuffdiff. 
in the KEAP1 gene and proteasome-mediated degradation of Nrf2 is impaired. ${ }^{18}$ However, it is reported that KEAP1 also has a degradation-independent inhibitory function on Nrf2. ${ }^{19}$ To address how RBM47 inhibits Nrf2 target gene expression in the absence of degradation machinery, we performed ChIP-qPCR analysis using the anti-Nrf2 antibody. We focused on GPX2, SRXN1 and HMOX1 as direct target genes of Nrf2 in A549 cells; ${ }^{2,20}$ expression of these genes was upregulated by shRBM47 (Supplementary Table S3 and Figure 3c). Strikingly, we found that the binding strength of Nrf2 to the $5^{\prime}$ promoter-flanking regions of GPX2 and SRXN1 loci, but not HMOX1 locus, was upregulated in A549-Luc-shRBM47 cells (Figure 3g). Enhanced Nrf2 binding to GPX2 and HMOX1 loci was also observed in another cell line (H441 cells) transfected with RBM47 siRNAs (Figure 3h). It should be noted that the percent input value of Nrf2 binding at the HMOX1 locus in A549-Luc cells was higher than that in H441 cells and that at the other target gene loci, suggesting a contribution of KEAP1 mutation and a difference in the affinity of Nrf2 binding to the loci, respectively.

TGF- $\beta$ is known to upregulate the expression of CDKN1A and MafK ${ }^{5,21}$ In the absence of RBM47, we found the enhanced induction of CDKN1A and MafK by TGF- $\beta$ in A549-Luc cells (Figure 3i). Taken together, these findings suggest that RBM47 inhibits Nrf2 activity in an integrated manner through targeting several key regulatory molecules, that is, KEAP1, CUL3, p21/CDKN1A and MafK. Upregulation of CDKN1A and MafK mRNAs by shRBM47 appears to be an indirect process because RBM47 does not bind to their mRNAs.

\section{RBM47 regulates oxygen consumption and drug resistance}

Nrf2 is known as one of the most important factors of cellular respiratory metabolism and drug resistance. ${ }^{7}$ We then examined the effect of RBM47 on cellular metabolism by measuring oxygen consumption rates (OCR). We found that the basal respiration rate was significantly upregulated in A549-Luc-shRBM47 cells (Figures $4 a$ and b). Decrease in OCR after oligomycin (an inhibitor of mitochondrial F0 ATPase) treatment was more significant in A549-Luc-shRBM47 cells than in A549-Luc-shCT cells, suggesting that ATP production was also upregulated by knockdown of RBM47. These findings indicated that mitochondrial respiration was suppressed by RBM47. Moreover, residual OCR was still higher in A549-Luc-shRBM47 cells in the presence of oligomycin, showing that the non-mitochondrial respiration rate was also upregulated in A549-Luc-shRBM47 cells. Finally, OCR level was not elevated after the addition of the carbonyl cyanide p-trifluoromethoxyphenyl hydrazine (FCCP) relative to before oligomycin treatment, suggesting that A549 cells fully utilize mitochondrial respiratory function and do not have a reserved capacity that is suppressed by RBM47. We also examined the effect of TGF- $\beta$ on cellular metabolism and found that TGF- $\beta$ increased the respiration rate, which was in agreement with the effect of shRBM47 (Figures $4 c$ and d).

We then examined the presence of side population cells in A549 cells, which have cancer stem cell-like properties and exhibit drug resistance. $^{22}$ The ratio of side population cells to the total cell population was determined by the number of cells that disappeared as a result of treatment with fumitremorgin C. Flow cytometry revealed that the fraction of side population cells was remarkably increased in A549-Luc-shRBM47 cells (Figure 4e). In contrast, TGF- $\beta$ rather decreased the fraction of side population cells (Figure $4 f$ ).

These results suggested that RBM47 represses respiratory metabolism and development of drug resistance. In addition, RBM47 might act as an effector downstream of TGF- $\beta$ signaling to repress respiratory metabolic activity, but the repression of side population cells by RBM47 is overcome by some other mechanism(s) regulated by TGF- $\beta$.

\section{Effect of RBM47 on tumor progression}

We found that cell growth was decreased in A549-Luc-shRBM47 cells compared with A549-Luc-shCT cells in vitro (Supplementary Figure S3a). However, when A549-Luc-shCT and A549-Luc-shRBM47 cells were subcutaneously injected into nude mice, the tumor volume was greater in mice injected with A549-Luc-shRBM47 cells than in mice injected with A549-Luc-shCT cells (Figures 5a and b and Supplementary Figures S3b and c). We also used another xenograft assay. A549-Luc-shCT and A549-Luc-shRBM47 cells were injected into the tail vein of nude mice, and lung metastasis was monitored by photon counting. In vivo imaging revealed that metastatic lesion was larger in nude mice injected with A549-shRBM47 cells than in nude mice injected with A549-Luc-shCT cells (Figures $5 \mathrm{c}$ and d). These results suggested that RBM47 suppressed tumor growth in vivo, which is in agreement with data obtained from the Kaplan-Meier analysis of lung adenocarcinoma patients (Figure 1d).

Taken together, our results suggest that RBM47 suppresses Nrf2 activity by upregulating KEAP1 and CUL3, and suppressing some Nrf2 activators (Figure 6), leading to the inhibition of tumor growth in vivo.

\section{DISCUSSION}

TGF- $\beta$ family cytokines execute pleiotropic functions in normal and transformed cells. TGF- $\beta$ family proteins and their downstream Smad proteins have been reported to posttranscriptionally regulate the function of RNAs. Davis et al. ${ }^{23}$ revealed that Smad proteins are recruited in the DROSHA microprocessor complex and regulate the maturation of miRNAs. TGF- $\beta$ has also been reported to regulate the expression of various miRNAs, including miR-34a, which suppresses the CCL22 production that is important for the recruitment of regulatory $T$ cells. ${ }^{24}$ In addition to its regulatory functions on miRNAs, TGF- $\beta$ is now known to regulate the expression of certain RNA-binding proteins. We have reported that the expression of epithelial splicing regulatory protein (ESRP) 1 and ESRP 2 was transcriptionally suppressed by TGF- $\beta$, and that they were involved in anti-epithelial-to-mesenchymal transition function through alternative splicing of various mRNAs, including fibroblast growth factor receptor 2 (FGFR2) mRNA. ${ }^{25}$ In the present study, we have shown that RBM47 inhibits Nrf2 activity to promote tumor growth in lung adenocarcinoma cells. Noteworthy, Rbm47 exhibited a much greater fold change upon TGF- $\beta$ treatment than Esrp2 in NMuMG cells (Supplementary Table S1). In general, the effect of shRBM47 on gene expression was comparable with that of TGF- $\beta$ stimulation (Supplementary Figure S4a). Therefore, RBM47 may partly exert its function as a downstream effector of TGF- $\beta$. It is also possible that RBM47 may regulate TGF- $\beta$ signaling pathway. However, some of the TGF- $\beta$ target genes were differentially regulated by RBM47, for example, induction of Snail and Slug by TGF- $\beta$ was attenuated in the absence of RBM47 (Supplementary Figure S4b).

Integrated molecular profiling of lung adenocarcinoma revealed that KEAP1 mutation was observed in $19 \%$ of the tumors. ${ }^{26}$ Functional analysis of 18 mutations in the KEAP1 gene performed by Hast et al. ${ }^{19}$ revealed that the G333C mutation found in A549 cells resulted in attenuated Nrf2 binding, while binding to CUL3 was reserved. We demonstrated that while shRBM47 downregulated KEAP1 and CUL3 proteins, it did not change the expression of Nrf2 protein. Nevertheless, enhanced Nrf2 binding to the genome and enhanced expression of target genes were induced by shRBM47. This observation strengthens the recent finding that some KEAP1 mutations retain inhibitory activity independent of Nrf2 degradation. ${ }^{19}$ It is possible that the mutant KEAP1 protein inhibits Nrf2 activity at the point of Nrf2 binding to the genome. This mode of inhibition might be target-dependent and cell type-dependent, because shRBM47 enhanced HMOX1 expression without significant change in Nrf2 binding to the 
a

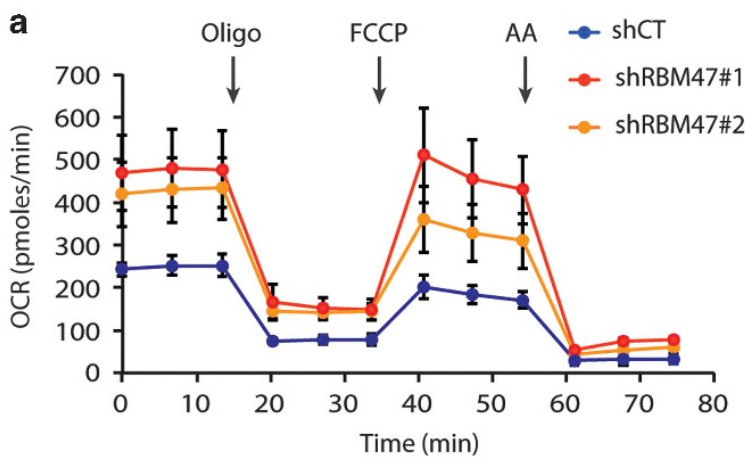

C

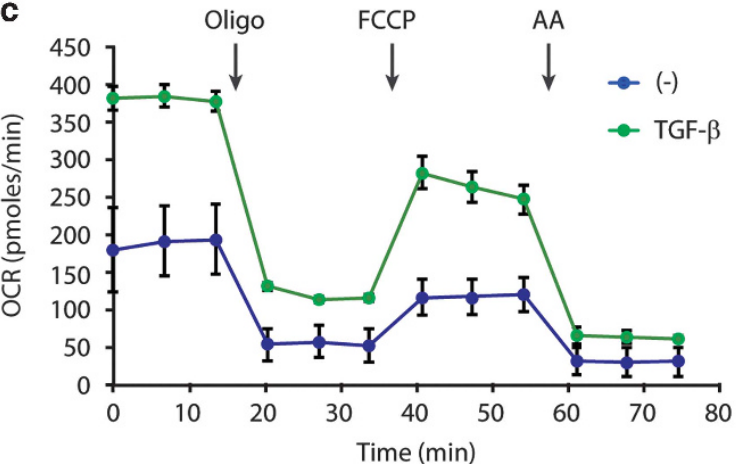

b

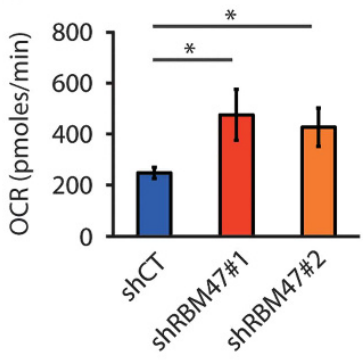

Basal respiration

d

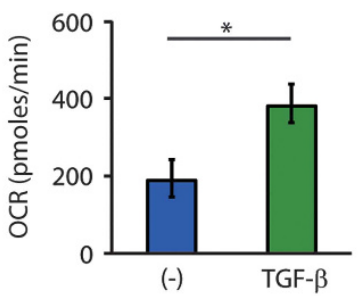

Basal respiration
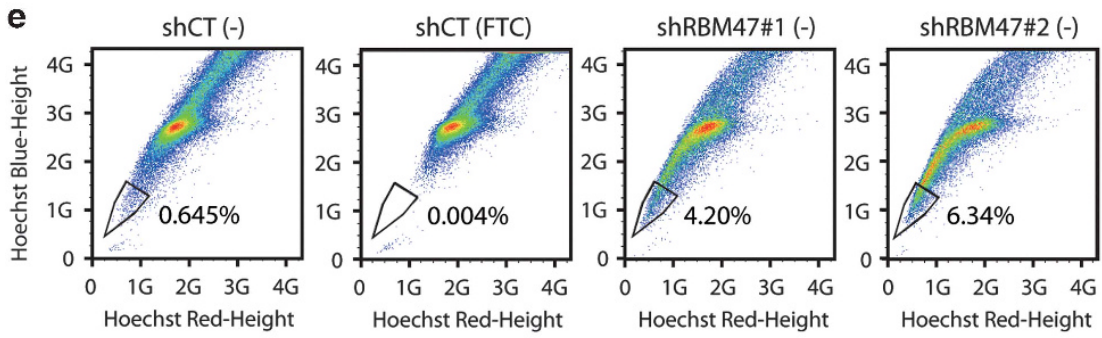

f
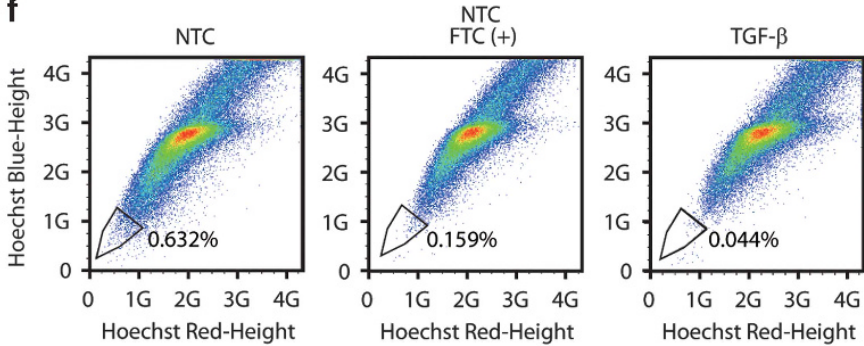

Figure 4. Knockdown of RBM47 results in Nrf2-related cellular biological responses. (a) The effects of shRBM47s on the oxygen consumption of A549 cells. OCRs in A549-Luc-shCT cells and A549-Luc-shRBM47 cells were examined using the XF Cell Mito-Stress test kit and XF24 extracellular flux analyzer. The final concentrations of oligomycin (Oligo) and antimycin A (AA) were $1 \mu \mathrm{m}$; the final concentration of FCCP was $16 \mu$. (b) Cumulative basal respiration quantities in A549-Luc-shCT cells and A549-Luc-shRBM47 cells. Values were calculated from the data in (a), and the difference of the significance was evaluated by $t$-test $\left({ }^{*} P<0.05\right)$. (c) The effect of TGF- $\beta$ on OCR in A549-Luc-shCT cells. OCR was measured as in (a). In (a) and (c), averages and standard deviations of the five biological replicates were shown for each condition. ${ }^{*} P<0.05$. (d) The effect of TGF- $\beta$ on cumulative basal respiration. Values were calculated as in (b). (e) The numbers of side population cells in A549-LucshCT and A549-Luc-shRBM47 cells were determined by flow cytometry. FTC: fumitremorgin C; (-): no treatment. Numbers indicate the fraction of the side population cells. (f) The effect of TGF- $\beta$ on the ratio of side population cells in A549-Luc-shCT cells was determined as in (e). For each analysis, cells were stimulated with $2.5 \mathrm{ng} / \mathrm{ml}$ of TGF- $\beta$ for $24 \mathrm{~h}$.

HMOX1 locus in A549 cells, while knockdown of RBM47 increased the Nrf2 binding to it in H441 cells (Figures $3 \mathrm{~g}$ and $\mathrm{h}$ ). In addition, shRBM47 minimally induced NQO1 (1.2-fold induction), a wellknown target of Nrf2, which was strongly expressed even in the presence of RBM47. This kind of target selectivity during the regulation of transcription factor activity has already been confirmed for Smad family proteins downstream of TGF- $\beta$, and was explained by the difference in the binding sequence and cobinding factors on the genome. ${ }^{15,27,28}$ Of note, unlike the present study using A549 cells, TGF- $\beta$ inhibited the oxidative stress response by Nrf2 through the induction of Maf family transcription factors in cooperation with Bach1 in mammary gland epithelial 
5006
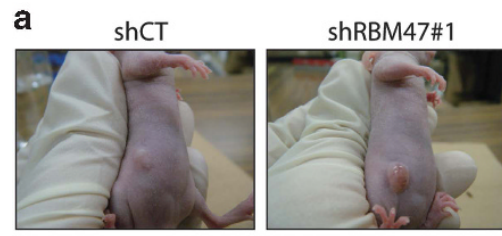

c

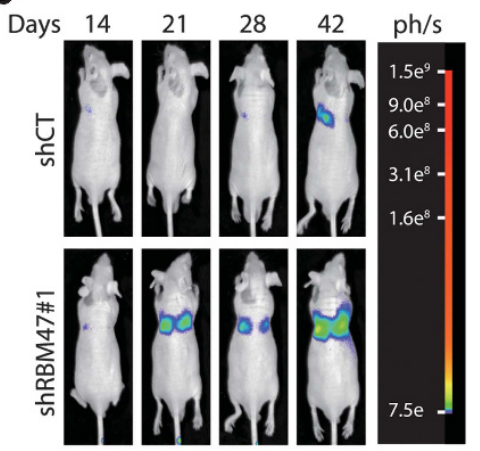

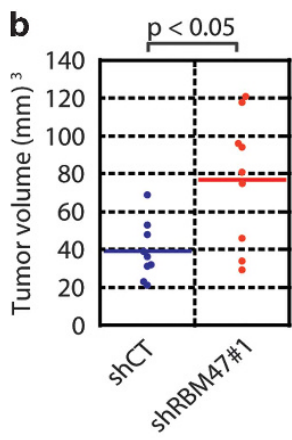

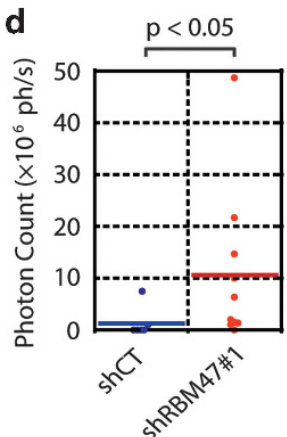

Figure 5. RBM47 suppresses tumor progression in vivo. (a) Photographs of the representative tumors xenografted with A549-Luc-shCT cells and A549-Luc-shRBM47\#1 cells. Cells were implanted subcutaneously into nude mice, and 24 days later, the tumor volume was measured. (b) Tumor volumes shown on a dot plot chart. $n=9$ for each condition. (c) Examples of the time-course change in luminescence from xenografted mice. A549-Luc-shCT cells and A549-LucshRBM47\#1 cells were injected into the tail vein of nude mice. The lung metastasis was monitored by photon counting after intraperitoneal administration of D-luciferin. (d) Photon counts shown by a dot plot chart. $n=6$ for shCT and $n=10$ for shRBM47\#1. The horizontal lines in the dot plots indicate average values in (b) and (d). ph/s: photon counts/second.

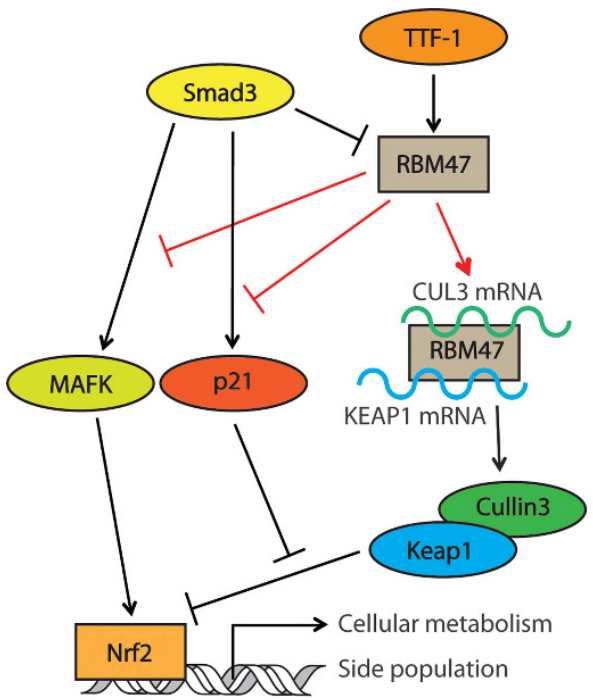

Figure 6. Mechanism of action of RBM47 through Nrf2 activity. RBM47 binds KEAP1 and CUL3 mRNAs to enhance their protein expression. RBM47 also transcriptionally (and indirectly) suppresses p21/CDKN1A and MafK expression both under unstimulated and TGF- $\beta$-stimulated conditions. CDKN1A suppression by RBM47 may result in enhanced KEAP1/CUL3-mediated inhibition of Nrf2.,5 Regulation of MafK expression may provide target and context dependency for the effects of both TGF- $\beta$ and RBM47 on Nrf2 functions. ${ }^{21}$ cells. ${ }^{21}$ Our results also suggested that the effect of TGF- $\beta$ on the number of side population cells appears to be independent of the effect of RBM47. These observations suggested the presence of regulatory machinery of Nrf2 on the genome, as well as a cell type-dependent difference in the effect of TGF- $\beta$ on Nrf2 activity. Taken together, our findings strengthen the importance of considering Nrf2-mediated transcriptional regulation not by the uniform on-and-off mechanism, but by the context-dependent and target-by-target mechanisms.

Exogenously expressed RBM47 reportedly binds to Nanog mRNA in mouse embryonic stem cells. ${ }^{29}$ In addition, the analysis of RBM47 function during zebrafish development revealed that RBM47 is involved in the Wnt signaling pathway. ${ }^{11}$ The inhibitory effect of RBM47 on brain metastasis of breast cancer through the suppression of Wnt signaling has been demonstrated, mainly using exogenously expressed RBM $47 .^{30}$ Their high-throughput sequencing and cross-linking immunoprecipitation analysis indicated that exogenous RBM47 binds to the 3'UTR of mRNAs and regulates alternative splicing, although a calculated binding motif was not identical to the 'GAUGAU' sequence determined by Ray et al., $^{31}$ and was almost a series of $U$ nucleotides. Our RIP-seq result showed that RBM47 binds to ATP5L, NDUFS6 and other mRNAs, which are related to cellular metabolism but not directly regulated by the Nrf2 pathway (Supplementary Table S4 and Supplementary Figure S4c). The expression levels of ATP5L and NDUFS6 mRNAs and proteins were slightly upregulated by shRBM47\#1 (Supplementary Figures S4d and e), suggesting the presence of multiple targets of RBM47. It is therefore possible that other phenotypes related to other targets of RBM47 may become more significant in some cellular contexts (for example, in the absence of Nrf2 activation).

Fossat et al. ${ }^{10}$ provided evidence of the regulation of $C$ to $U$ RNA editing by RBM47 through APOBEC1 in the nucleus. Their findings suggested an important mode of action of RBM47 through the editing of the $3^{\prime}$ UTR of target RNAs. As a result, it is possible that RBM47 regulates the stability or translation of RNAs either positively or negatively, although the tumor-promoting aspect of RNA editing by the APOBEC family has mainly been demonstrated. ${ }^{32,33}$ Unfortunately, our limited sequencing depth of RNA-seq data and genomic data from A549 cells did not produce any evidence that editing of KEAP1 and CUL3 RNA is induced by RBM47. In addition, we cannot exclude the possibility that cytoplasmic RBM47 has some functions different from nuclear RBM47. Further analysis of the data with improved sequencing depth will be required to elucidate the precise mechanism by which bound RNAs are regulated by RBM47.

\section{MATERIALS AND METHODS}

Reagents and antibodies

Recombinant TGF- $\beta$ (TGF- $\beta 3$ ) was purchased from R\&D systems (Minneapolis, MN, USA). The following antibodies were used: mouse anti-a-tubulin (DM1A; Sigma-Aldrich, St Louis, MO, USA), mouse anti-TTF-1 (8G7G3/1; Novus Biologicals, Littleton, CO, USA), rabbit anti-RBM47 (Sigma-Aldrich, HPA006347), anti-Nrf2 (EP1808Y; Abcam, Cambridge, UK), anti-KEAP1 (10503-2-AP; Proteintech, Chicago, IL, USA), anti-CUL3 (Abcam, EPR3196Y), anti-HDAC1 (2E10; Millipore, Billerica, MA, USA), anti-ATP5L (Abcam, ab126181) and anti-NDUFS6 (Abcam, ab156099).

\section{Cell culture}

A549 cells were from Cell Resource Center for Biomedical Research, Institute of Development, Aging, and Cancer, Tohoku University. NCl-H441 (H441) cells were from American Type Culture Collection (Manassas, VA, USA). Cell authentication was not performed by ourselves. A549 and HEK293T cells were maintained in Dulbecco's modified Eagle's medium (\#11965; Thermo Fisher Scientific, Waltham, MA, USA) supplemented with $10 \%$ fetal bovine serum, $100 \mathrm{U} / \mathrm{ml}$ penicillin $\mathrm{G}$ and $100 \mu \mathrm{g} / \mathrm{ml}$ streptomycin. $\mathrm{H} 441$ cells were maintained in RPMI 1640 (\#11875; Thermo Fisher Scientific) supplemented with $10 \%$ fetal bovine serum, $100 \mathrm{U} / \mathrm{ml}$ penicillin $\mathrm{G}$, and $100 \mu \mathrm{g} / \mathrm{ml}$ streptomycin. Cells were grown in a humidified atmosphere with $5 \% \mathrm{CO}_{2}$ 
at $37^{\circ} \mathrm{C}$. Absence of mycoplasma contamination was confirmed by e-Myco VALiD Mycoplasma PCR detection kit using input genomic DNAs in Figures $3 \mathrm{~g}$ and $\mathrm{h}$ (CosmoBio, Tokyo, Japan).

\section{Chromatin immunoprecipitation}

A549-Luc and H441 cells were cultured in $10-\mathrm{cm}$ plates, and ChIP was performed as described. ${ }^{34}$ All samples were run in technical triplicates, and the results were averaged. The experiments were performed twice with essentially the same results and the representative data were shown.

\section{ChIP-seq data}

TTF-1- and Smad3-binding regions at the RBM47 locus were determined using our published ChIP-seq data (GEO accession no: GSE51510). ${ }^{15}$

\section{RNA interference using siRNAs}

Pre-designed Stealth Select siRNAs (siTTF-1\#1 (HSS144278), siTTF-1\#2 (HSS111041), siSmad3\#1 (HSS106252), siSmad3\#2 (HSS180970), siRBM47\#1 (HSS122967), siRBM47\#2 (10620312) and control siRNAs (12935-200 for H441 cells and 12935-112 for A549 cells)) were purchased from Thermo Fisher Scientific. siRNAs were introduced into $\mathrm{H} 441$ cells or A549 cells through the reverse transfection method using Lipofectamine RNAiMAX reagent (Thermo Fisher Scientific) according to the manufacturer's instructions. The final concentration of siRNA in the culture medium was $50 \mathrm{~nm}$.

Quantitative reverse transcription-polymerase chain reaction Total RNAs were extracted using TriPure (Roche Diagnostics, Basel, Switzerland). First-strand CDNA was synthesized as described previously. ${ }^{9}$ qRT-PCR analysis was performed using FastStart Universal SYBR Green Master Mix with ROX (Roche Diagnostics), and the ABI PRISM 7000 Sequence Detection System or the StepONE Plus real time PCR system (Thermo Fisher Scientific). All samples were run in two technical replicates, and the results were averaged. The experiments were performed at least twice, except for the evaluation in the panel of cell lines (Figure 1c), with essentially the same results and the representative data were shown.

\section{Promoter-reporter constructs and cDNA constructs}

Adenoviral expression vectors of LacZ and TTF-1 were prepared as described. ${ }^{16}$ The $5^{\prime}$ flank of the human RBM47 promoter region (-1950 to +50 ) was PCR-amplified from the genomic DNA of H441 cells using PrimeSTAR GXL DNA polymerase (Takara Bio, Shiga, Japan) with two sets of primers. Equal amounts of the isolated PCR products were mixed, denatured, annealed at room temperature and ligated into the HindllIEcoRI restriction site of pGL4.10 (Promega, Fitchburg, WI, USA). Combinations of the primers used for amplification were: Forward 1: AGCT TACACAGCTAGGAAGTCACCTACAACATCTT; Reverse 1: CATTAAGCATAGC AGATGGGTGATTGCTGGG; Forward 2: TACACAGCTAGGAAGTCACCTACAA CATCTT; Reverse 2: AATT CATTAAGCATAGCAGATGGGTGATTGCTGGG.

\section{Dual-luciferase assay}

A549 and H441 cells cultured in 12-well plates were transfected with RBM47 promoter luciferase constructs. Total amounts of transfected DNA were adjusted to the same quantities with an empty vector. For normalization, pGL4.75-SV40-hRluc was co-transfected. Cells were harvested and assayed for luciferase activity using the Dual-Luciferase Reporter Assay System (Promega) and Mithras LB-940 (Berthold technologies, Bad Wildbad, Germany). The experiments were performed at least twice with essentially the same results and the representative data were shown.

\section{Immunoblotting}

Lysis buffer (1\% NP-40, $150 \mathrm{~mm} \mathrm{NaCl}, 20 \mathrm{~mm}$ Tris- $\mathrm{HCl}$ (pH 7.5) and cOmplete EDTA-free protease inhibitor cocktail from Roche Diagnostics) was used for cell lysis. The nucleus and cytoplasm were fractionated using the NE-PER Nuclear and Cytoplasmic Extraction Kit (Thermo Fisher Scientific) according to the manufacturer's instructions. The same amount of the proteins was loaded on the gels in each fraction. Sodium dodecyl sulfate gel electrophoresis and immunoblotting were performed as described, ${ }^{35}$ using a LAS-4000 lumino-image analyzer (GE Healthcare, Buckinghamshire, UK). The experiments were performed at least twice with essentially the same results and the representative data were shown.
Lentiviral luciferase and shRNA expression

A549 cells were sequentially infected with lentiviral vectors carrying EF promoter-driven luciferase and $\mathrm{H} 1$ promoter-driven shRNAs to establish A549-Luc cells stably expressing both luciferase and gene-specific shRNAs, as described. ${ }^{36}$ The following oligonucleotides were used to construct the shRNA vectors: shRBM47\#1, Forward: GATCCCC GGGCTCAGTATTCCATGTTTC ACGTGTGCTGTCCGT GAAACATGGAATACTGAGCCC TाTाTGGAAAT, Reverse: CT AGATTTCCAAAAA GGGCTCAGTATTCCATGTTTC ACGGACAGCACACGTGAAACAT GGAATACTGAGC CCGGG; shRBM47\#2, Forward: GATCCCC GGATCTCTCCTTAA GCCAACA ACGTGTGCTGTCCGT TGTTGGCTTAAGGAGAGATCC TाTाTGGAAAT, Reverse: CTAGATTTCCAAAAA GGATCTCTCCTTAAGCCAACA ACGGACAGCACAC GTTGTTGGCTTAAGGAGAGAT CCGGG.

\section{RNA-seq and data analysis}

cDNA libraries were prepared through the sequential use of the RNeasy Mini Kit with On-Column DNase Digestion Set (QIAGEN, Venlo, Netherlands), Dynabeads mRNA DIRECT Purification Kit and Ion Total RNA-Seq Kit v2 (Thermo Fisher Scientific) and were sequenced and analyzed as in RIP-seq.

Gene ontology analysis

Gene set enrichment analysis was performed using GSEA. ${ }^{37}$

\section{Flow cytometry}

Flow cytometric analysis was performed as described. ${ }^{38,39}$ Cells were analyzed using a MoFlo Astrios cell sorter (Beckman Coulter, Brea, CA, USA). Gate for side population cells was defined as diminished region in the presence of fumitremorgin C. Data were analyzed using Flow Jo software (Treestar, Ashland, OR, USA).

\section{Tumor xenograft assay and in vivo bioluminescence imaging} analysis

All animal experiments were performed in accordance with the policies of the animal ethics committee of the University of Tokyo. Four-week-old male Balb/c nude mice were injected subcutaneously with $1.0 \times 10^{7}$ A549-LucshCT cells or A549-Luc-shRBM47\#1 cells. Investigators were not blinded during the tumor injection and outcome assessment in the subcutaneous xenograft trial. Tumor volume was measured and calculated using the following formula: ((major axis $\left.) \times(\text { minor axis })^{2}\right) / 2$. In a lung metastasis model, 4-week-old male Balb/c nude mice were tail-vein-injected with $3.0 \times 10^{7}$ A549-Luc-shCT cells or A549-Luc-shRBM47\#1 cells. Whole-body optical imaging was performed by photon counting (LB983, Berthold Technologies) after intraperitoneal injection of D-luciferin potassium salt (Wako Chemicals, Tokyo, Japan). No statistical methods were used for sample size estimation. Mice were randomly divided into groups. In the lung metastasis trial, investigators were blinded during the tumor injection and not informed as to the expected results during image acquisition. Mice died within hours after tumor cell injection were excluded from the evaluation. The criteria were determined in advance.

\section{RIP-seq and data analysis}

A549 cells were cultured in 10-cm plates and the RIP assay was performed using the Magna RIP RNA-Binding Protein Immunoprecipitation Kit (Millipore), following the manufacturer's protocols. Rabbit control IgG and anti-SNRNP70 provided by the kit were used as controls. Libraries were made from RIP samples using lon Total RNA-Seq Kit v2. High-throughput sequencing of the CDNA library was performed using lon PROTON, Ion PI Template OT2 200 Kit v3 and lon PI Sequencing 200 Kit v3..$^{15}$ Sequence reads were aligned against the human reference transcriptome (NCBI Build 37, hg19) using TopHat2. Expression levels were calculated using the cuffdiff function of Cufflinks.

\section{Flux analyzer}

OCRs were measured with the Seahorse Biosciences extracellular flux analyzer (XF24; North Billerica, MA, USA) with an XF cell mitochondrial stress kit. A total of $1.0 \times 10^{5}$ cells were seeded per well in growth medium (Dulbecco's modified Eagle's medium with $10 \%$ fetal bovine serum) and incubated for $12 \mathrm{~h}$ at $37^{\circ} \mathrm{C}$ and $5 \% \mathrm{CO}_{2}$ before measurement. After incubation, the medium was changed to assay medium, which contains Dulbecco's modified Eagle's medium (Sigma D5030), $1.85 \mathrm{~g} / \mathrm{l} \mathrm{NaCl}, 2 \mathrm{~mm}$ I-glutamine, $1 \mathrm{~mm}$ sodium pyruvate and $50 \mathrm{~mm}$ glucose. After $1 \mathrm{~h}$ incubation with atmospheric $\mathrm{CO}_{2}$ level at $37^{\circ} \mathrm{C}$, basal respiration rates 
were measured periodically. ATP production rates were measured after oligomycin injection (final $1 \mu \mathrm{M}$ ). Maximal respiration rates were measured after carbonyl cyanide p-trifluoromethoxyphenyl hydrazine (FCCP) injection (final $12 \mu \mathrm{M}$ ), followed by the measurement of non-mitochondrial respiration rates after antimycin $A$ injection (final $1 \mu \mathrm{M}$ ). The experiments were performed at least twice with essentially the same results and the representative data were shown.

\section{Accession numbers}

Raw RNA-seq and RIP-seq data are available at GEO (GSE61910).

\section{Statistical analysis}

Student's $t$-test was used for two-sample analyses. The Mann-WhitneyWilcoxon test (Mann-Whitney $\mathrm{U}$ test) of the R program (http://www.rproject.org/) was used for in vivo experimental data. No statistical methods were used for sample size selection.

\section{CONFLICT OF INTEREST}

The authors declare no conflict of interest.

\section{ACKNOWLEDGEMENTS}

We are grateful to Dr Hiroyuki Aburatani, Shuichi Tsutsumi and Aya Nonaka (The University of Tokyo) for the ChIP-seq data and advice regarding RIP-seq analysis, and to Dr Hiroyuki Suzuki (Tsukuba University) for discussion and advice. We also thank Akiyoshi Komuro for a lentiviral luciferase expression plasmid and Keiko Yuki for technical assistance, and members of the Miyazono laboratory for discussion and advice. This work was supported by KAKENHI grants-in-aid for scientific research in Innovative Area (Integrative Research on Cancer Microenvironment Network, (22112002) to KM), Scientific Research (C) ((15K06831) to DK) and (S) ((15H05774) to KM), a Research Activity Start-up grant ((24890039), KI) from the Ministry of Education, Culture, Sports, Science and Technology of Japan (MEXT); and a grant from the Japan Agency for Medical Research and Development (AMED) (15ak0101001h0005 to DK). This study was performed as a research program of the Project for Development of Innovative Research on Cancer Therapeutics (P-Direct), AMED; MM is supported by the Kanae Foundation for Research Abroad and the ITO Genboku and SAGARA Chian Memorial Scholarship; TS is supported by a grant from the Japan Society for the Promotion of Science (JSPS).

\section{AUTHOR CONTRIBUTIONS}

TS performed in vitro experiments. TS, SS and SE performed and YM assisted with the in vivo experiments. TS, KI and DK performed high-throughput sequencing. MM developed the protocol for RNA-seq data analysis. TS, KI, KM and DK designed experiments and analyzed data. TS, KM and DK wrote the manuscript.

\section{REFERENCES}

1 Kobayashi A, Kang Ml, Okawa H, Ohtsuji M, Zenke Y, Chiba T et al. Oxidative stress sensor Keap1 functions as an adaptor for Cul3-based E3 ligase to regulate proteasomal degradation of Nrf2. Mol Cell Biol 2004; 24: 7130-7139.

2 Singh A, Ling G, Suhasini AN, Zhang P, Yamamoto M, Navas-Acien A et al. Nrf2-dependent sulfiredoxin-1 expression protects against cigarette smokeinduced oxidative stress in lungs. Free Radic Biol Med 2009; 46: 376-386.

3 Zhang $\mathrm{P}$, Singh A, Yegnasubramanian S, Esopi D, Kombairaju P, Bodas $M$ et al. Loss of Kelch-like ECH-associated protein 1 function in prostate cancer cells causes chemoresistance and radioresistance and promotes tumor growth. Mol Cancer Ther 2010; 9: 336-346.

4 Chen W, Sun Z, Wang XJ, Jiang T, Huang Z, Fang D et al. Direct interaction between Nrf2 and p21(Cip1/WAF1) upregulates the Nrf2-mediated antioxidant response. Mol Cell 2009; 34: 663-673.

5 Oshimori N, Oristian D, Fuchs E. TGF- $\beta$ promotes heterogeneity and drug resistance in squamous cell carcinoma. Cell 2015; 160: 963-976.

6 Mitsuishi Y, Taguchi K, Kawatani Y, Shibata T, Nukiwa T, Aburatani H et al. Nrf2 redirects glucose and glutamine into anabolic pathways in metabolic reprogramming. Cancer Cell 2012; 22: 66-79.

7 Hayes JD, Ashford ML. Nrf2 orchestrates fuel partitioning for cell proliferation. Cell Metab 2012; 16: 139-141.

8 Roberts $A B$, Wakefield LM. The two faces of transforming growth factor $\beta$ in carcinogenesis. Proc Natl Acad Sci USA 2003; 100: 8621-8623.
9 Arase M, Horiguchi K, Ehata S, Morikawa M, Tsutsumi S, Aburatani $\mathrm{H}$ et al. Transforming growth factor- $\beta$-induced IncRNA-Smad7 inhibits apoptosis of mouse breast cancer JygMC(A) cells. Cancer Sci 2014; 105: 974-982.

10 Fossat N, Tourle K, Radziewic T, Barratt K, Liebhold D, Studdert JB et al. C to U RNA editing mediated by APOBEC1 requires RNA-binding protein RBM47. EMBO Rep 2014; 15: 903-910.

11 Guan R, El-Rass S, Spillane D, Lam S, Wang Y, Wu J et al. rbm47, a novel RNA binding protein, regulates zebrafish head development. Dev Dyn 2013; 242: 1395-1404.

12 Gyorffy B, Lanczky A, Eklund AC, Denkert C, Budczies J, Li Q et al. An online survival analysis tool to rapidly assess the effect of 22,277 genes on breast cancer prognosis using microarray data of 1,809 patients. Breast Cancer Res Treat 2010; 123: 725-731.

13 Gyorffy B, Surowiak P, Budczies J, Lanczky A. Online survival analysis software to assess the prognostic value of biomarkers using transcriptomic data in non-smallcell lung cancer. PLoS One 2013; 8: e82241.

14 Yatabe Y, Mitsudomi T, Takahashi T. TTF-1 expression in pulmonary adenocarcinomas. Am J Surg Pathol 2002; 26: 767-773.

15 Isogaya K, Koinuma D, Tsutsumi S, Saito RA, Miyazawa K, Aburatani H et al. A Smad3 and TTF-1/NKX2-1 complex regulates Smad4-independent gene expression. Cell Res 2014; 24: 994-1008.

16 Saito RA, Watabe T, Horiguchi K, Kohyama T, Saitoh M, Nagase T et al. Thyroid transcription factor- 1 inhibits transforming growth factor- $\beta$-mediated epithelialto-mesenchymal transition in lung adenocarcinoma cells. Cancer Res 2009; 69: 2783-2791.

17 Malhotra D, Portales-Casamar E, Singh A, Srivastava S, Arenillas D, Happel C et al. Global mapping of binding sites for Nrf2 identifies novel targets in cell survival response through ChIP-Seq profiling and network analysis. Nucleic Acids Res 2010; 38: $5718-5734$.

18 Singh A, Misra V, Thimmulappa RK, Lee H, Ames S, Hoque MO et al. Dysfunctional KEAP1-NRF2 interaction in non-small-cell lung cancer. PLoS Med 2006; 3: e420.

19 Hast BE, Cloer EW, Goldfarb D, Li H, Siesser PF, Yan F et al. Cancer-derived mutations in KEAP1 impair NRF2 degradation but not ubiquitination. Cancer Res 2014; 74: 808-817.

20 Singh A, Rangasamy T, Thimmulappa RK, Lee H, Osburn WO, Brigelius-Flohe R et al. Glutathione peroxidase 2, the major cigarette smoke-inducible isoform of GPX in lungs, is regulated by Nrf2. Am J Respir Cell Mol Biol 2006; 35: 639-650.

21 Okita Y, Kamoshida A, Suzuki H, Itoh K, Motohashi H, Igarashi K et al. Transforming growth factor- $\beta$ induces transcription factors MafK and Bach1 to suppress expression of the heme oxygenase-1 gene. J Biol Chem 2013; 288: 20658-20667.

22 Sung JM, Cho HJ, Yi H, Lee CH, Kim HS, Kim DK et al. Characterization of a stem cell population in lung cancer A549 cells. Biochem Biophys Res Commun 2008; 371: 163-167.

23 Davis BN, Hilyard AC, Lagna G, Hata A. SMAD proteins control DROSHA-mediated microRNA maturation. Nature 2008; 454: 56-61.

24 Yang P, Li QJ, Feng Y, Zhang Y, Markowitz GJ, Ning S et al. TGF- $\beta$-miR-34a-CCL22 signaling-induced Treg cell recruitment promotes venous metastases of HBVpositive hepatocellular carcinoma. Cancer Cell 2012; 22: 291-303.

25 Horiguchi K, Sakamoto K, Koinuma D, Semba K, Inoue A, Inoue S et al. TGF- $\beta$ drives epithelial-mesenchymal transition through $\delta \mathrm{EF} 1$-mediated downregulation of ESRP. Oncogene 2012; 31: 3190-3201.

26 Cancer Genome Atlas Research N, Comprehensive molecular profiling of lung adenocarcinoma. Nature 2014; 511: 543-550.

27 Morikawa M, Koinuma D, Miyazono K, Heldin CH. Genome-wide mechanisms of Smad binding. Oncogene 2013; 32: 1609-1615.

28 Koinuma D, Tsutsumi S, Kamimura N, Taniguchi H, Miyazawa K, Sunamura M et al. Chromatin immunoprecipitation on microarray analysis of Smad2/3 binding sites reveals roles of ETS1 and TFAP2A in transforming growth factor $\beta$ signaling. Mol Cell Biol 2009; 29: 172-186.

29 Yeganeh M, Seyedjafari E, Kamrani FA, Ghaemi N. RNA-binding protein Rbm47 binds to Nanog in mouse embryonic stem cells. Mol Biol Rep 2013; 40: 4391-4396.

30 Vanharanta S, Marney CB, Shu W, Valiente M, Zou Y, Mele A et al. Loss of the multifunctional RNA-binding protein RBM47 as a source of selectable metastatic traits in breast cancer. elife 2014. e02734.

31 Ray D, Kazan H, Cook KB, Weirauch MT, Najafabadi HS, Li X et al. A compendium of RNA-binding motifs for decoding gene regulation. Nature 2013; 499: 172-177.

32 Valdmanis PN, Roy-Chaudhuri B, Kim HK, Sayles LC, Zheng Y, Chuang CH et al. Upregulation of the microRNA cluster at the Dlk1-Dio3 locus in lung adenocarcinoma. Oncogene 2015; 34: 94-103.

33 Petit V, Guetard D, Renard M, Keriel A, Sitbon M, Wain-Hobson S et al. Murine APOBEC1 is a powerful mutator of retroviral and cellular RNA in vitro and in vivo. $J \mathrm{Mol}$ Biol 2009; 385: 65-78.

34 Mizutani A, Koinuma D, Tsutsumi S, Kamimura N, Morikawa M, Suzuki HI et al. Cell type-specific target selection by combinatorial binding of $S m a d 2 / 3$ proteins and hepatocyte nuclear factor $4 a$ in HepG2 cells. J Biol Chem 2011; 286: 29848-29860. 
RBM47 inhibits Nrf2 activity in lung cancer cells

T Sakurai et al

35 Koinuma D, Shinozaki M, Nagano Y, Ikushima H, Horiguchi K, Goto K et al. RB1CC1 protein positively regulates transforming growth factor- $\beta$ signaling through the modulation of Arkadia E3 ubiquitin ligase activity. J Biol Chem 2011; 286: 32502-32512.

36 Shirai YT, Ehata S, Yashiro M, Yanagihara K, Hirakawa K, Miyazono K. Bone morphogenetic protein-2 and -4 play tumor suppressive roles in human diffuse-type gastric carcinoma. Am J Pathol 2011; 179: 2920-2930.

37 Subramanian A, Tamayo P, Mootha VK, Mukherjee S, Ebert BL, Gillette MA et al Gene set enrichment analysis: a knowledge-based approach for interpreting genome-wide expression profiles. Proc Natl Acad Sci USA 2005; 102: 15545-15550.

38 Ehata S, Johansson E, Katayama R, Koike S, Watanabe A, Hoshino Y et al. Transforming growth factor- $\beta$ decreases the cancer-initiating cell population within diffuse-type gastric carcinoma cells. Oncogene 2011; 30: 1693-1705.
39 Katayama R, Koike S, Sato S, Sugimoto Y, Tsuruo T, Fujita N. Dofequidar fumarate sensitizes cancer stem-like side population cells to chemotherapeutic drugs by inhibiting ABCG2/BCRP-mediated drug export. Cancer Sci 2009; 100: 2060-2068.

(c) (i) (2) This work is licensed under a Creative Commons Attributioncc) NonCommercial-ShareAlike 4.0 International License. The images or other third party material in this article are included in the article's Creative Commons license, unless indicated otherwise in the credit line; if the material is not included under the Creative Commons license, users will need to obtain permission from the license holder to reproduce the material. To view a copy of this license, visit http:// creativecommons.org/licenses/by-nc-sa/4.0/

Supplementary Information accompanies this paper on the Oncogene website (http://www.nature.com/onc) 